\title{
Computer vision technology for the alignment monitoring of SRF cryomodules
}

FERMILAB-POSTER-20-030-TD

\section{ALIGNMENT MONITORING STRATEGY}

- Two cameras per side are installed on the external flange to monitor the relative position of the components in the cryomodule

- Each cavity in the cryomodule is equipped with 4 targets
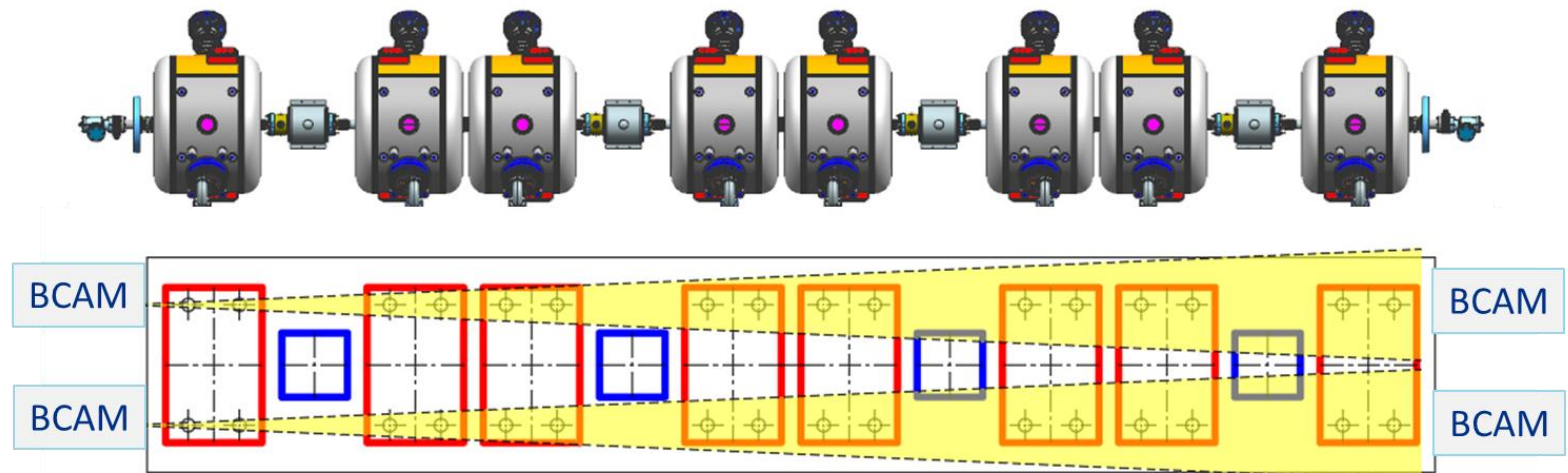

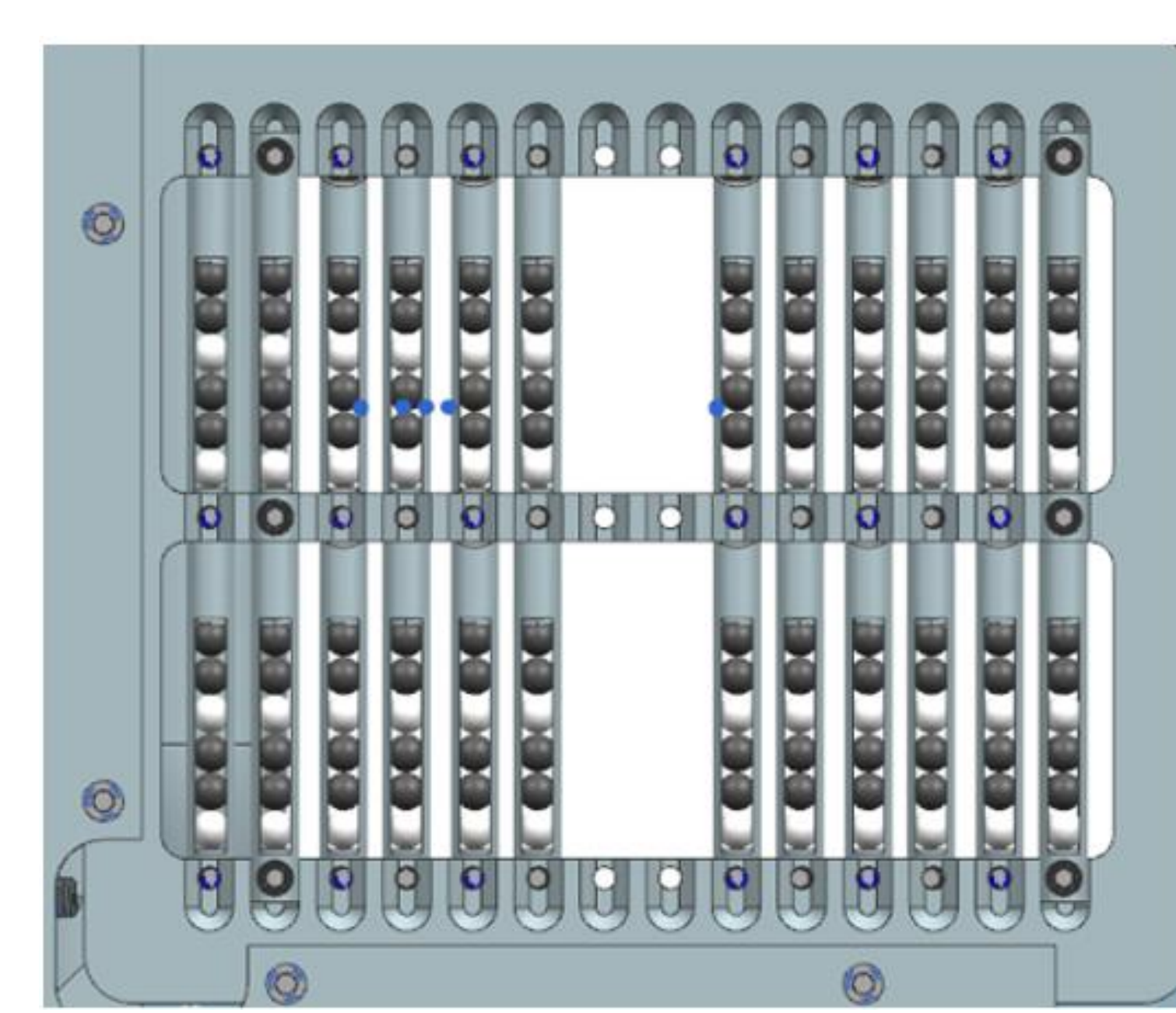

Targets transverse view model vs. camera

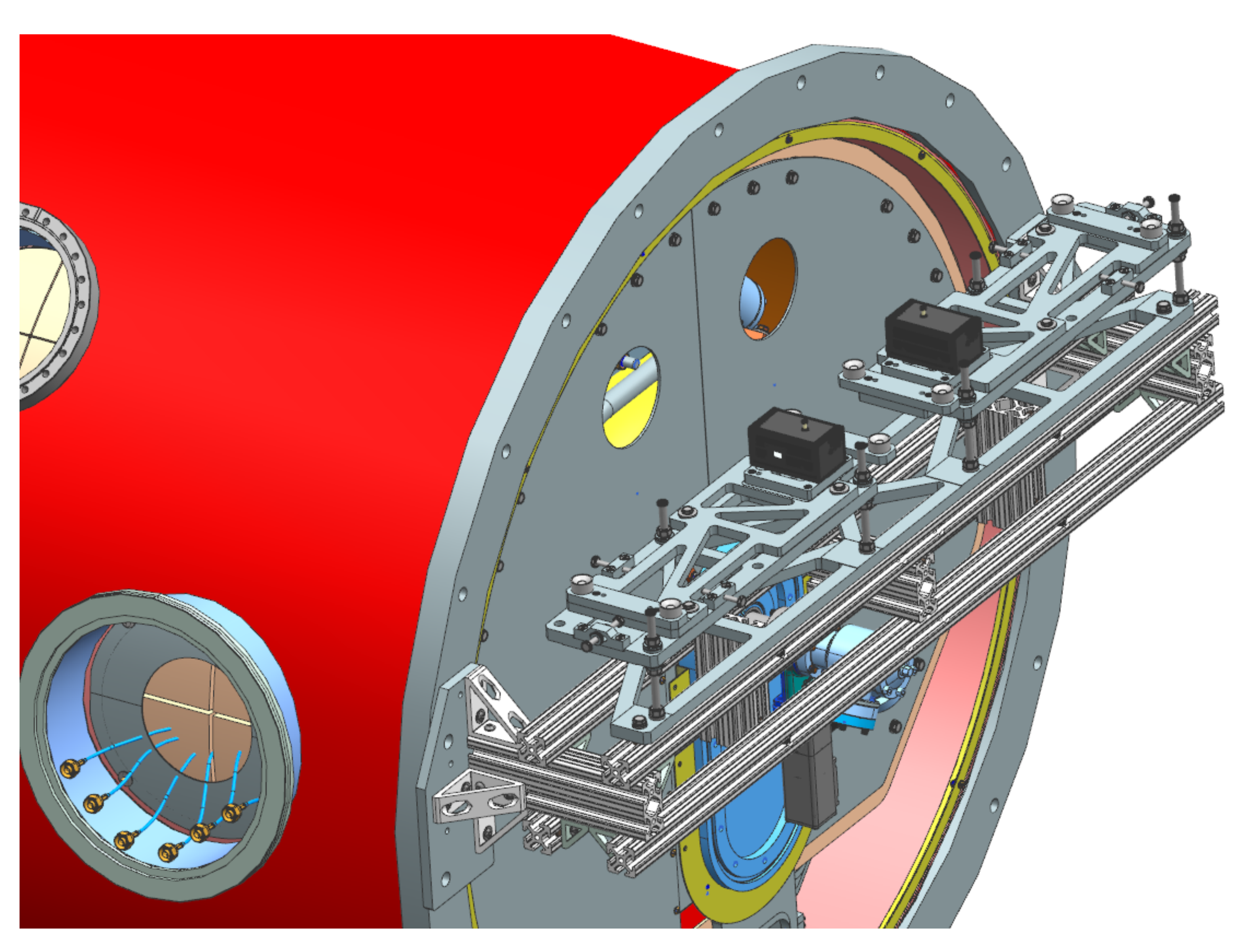

\section{COLDMASS INSERTION}

- Two cameras attached on the thermal shielding

- Two cameras installed at the ground and used as a reference
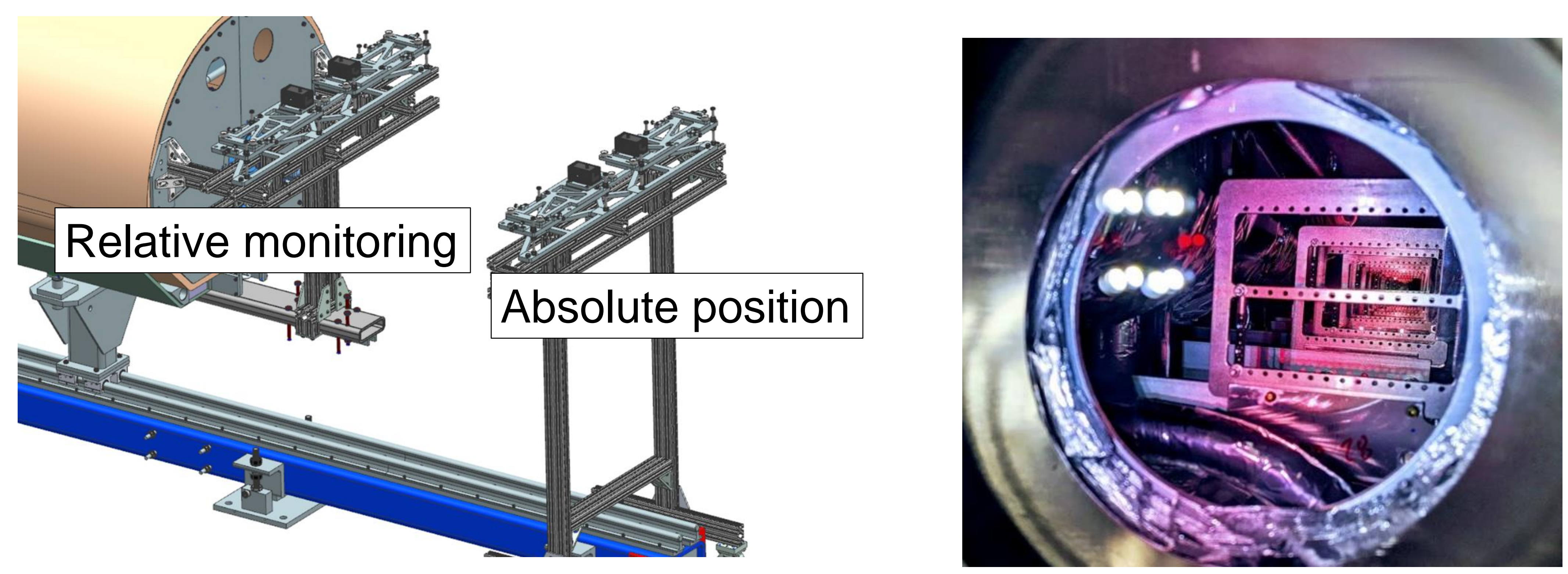

Targets assembly from viewport

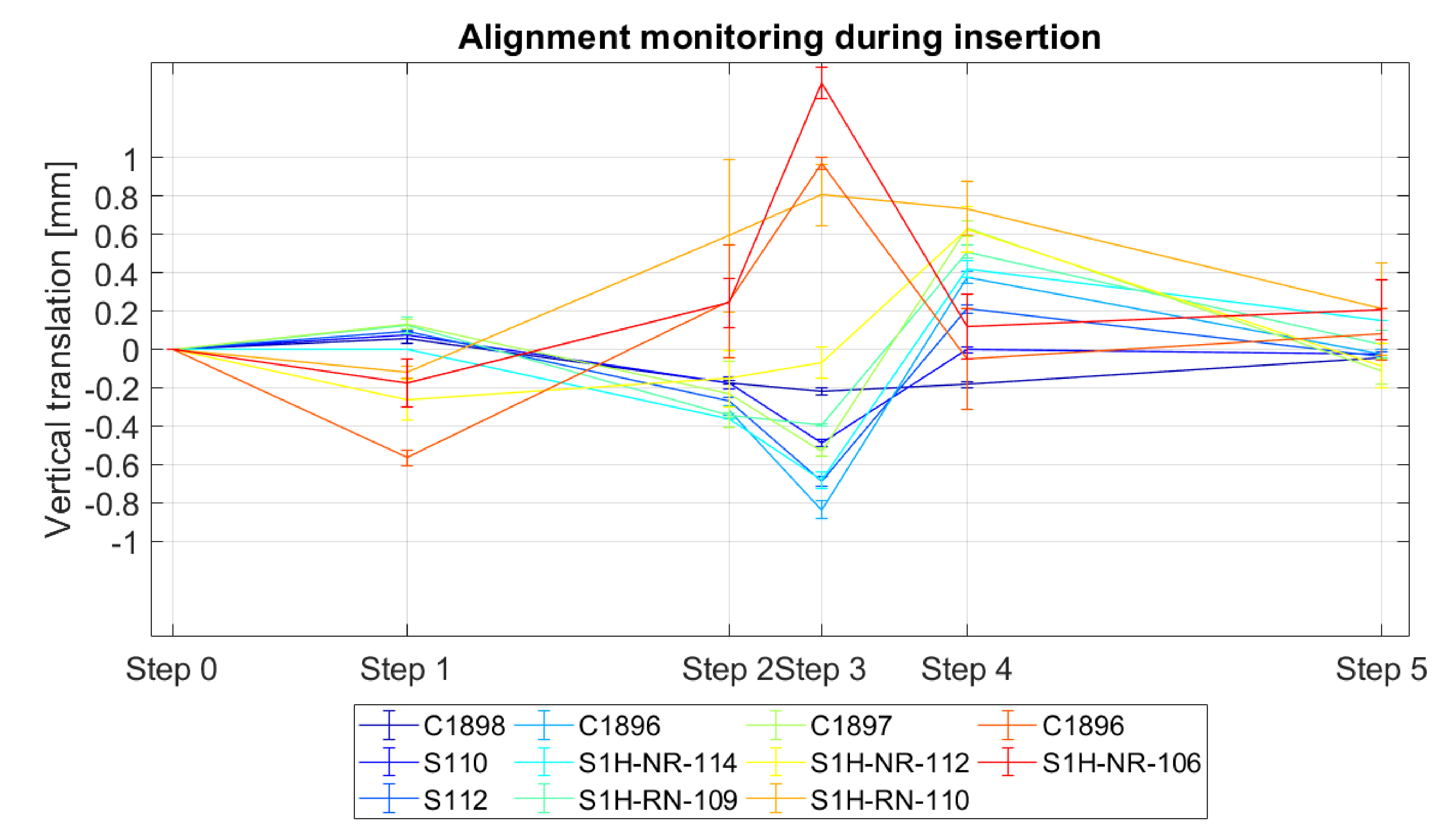

\section{COLDMASS INSERTION STEPS}

Step 0: Cavities and solenoids aligned employing laser trackers techniques. Strongback held by eight treaded supports

Step 1- 4: Insertion of the coldmass assembly into the vacuum vessel

Ste 5: Coldmass fully inserted and centered (aligned) with reference to the vacuum vessel by repositioning the strongback on eight threaded supports.

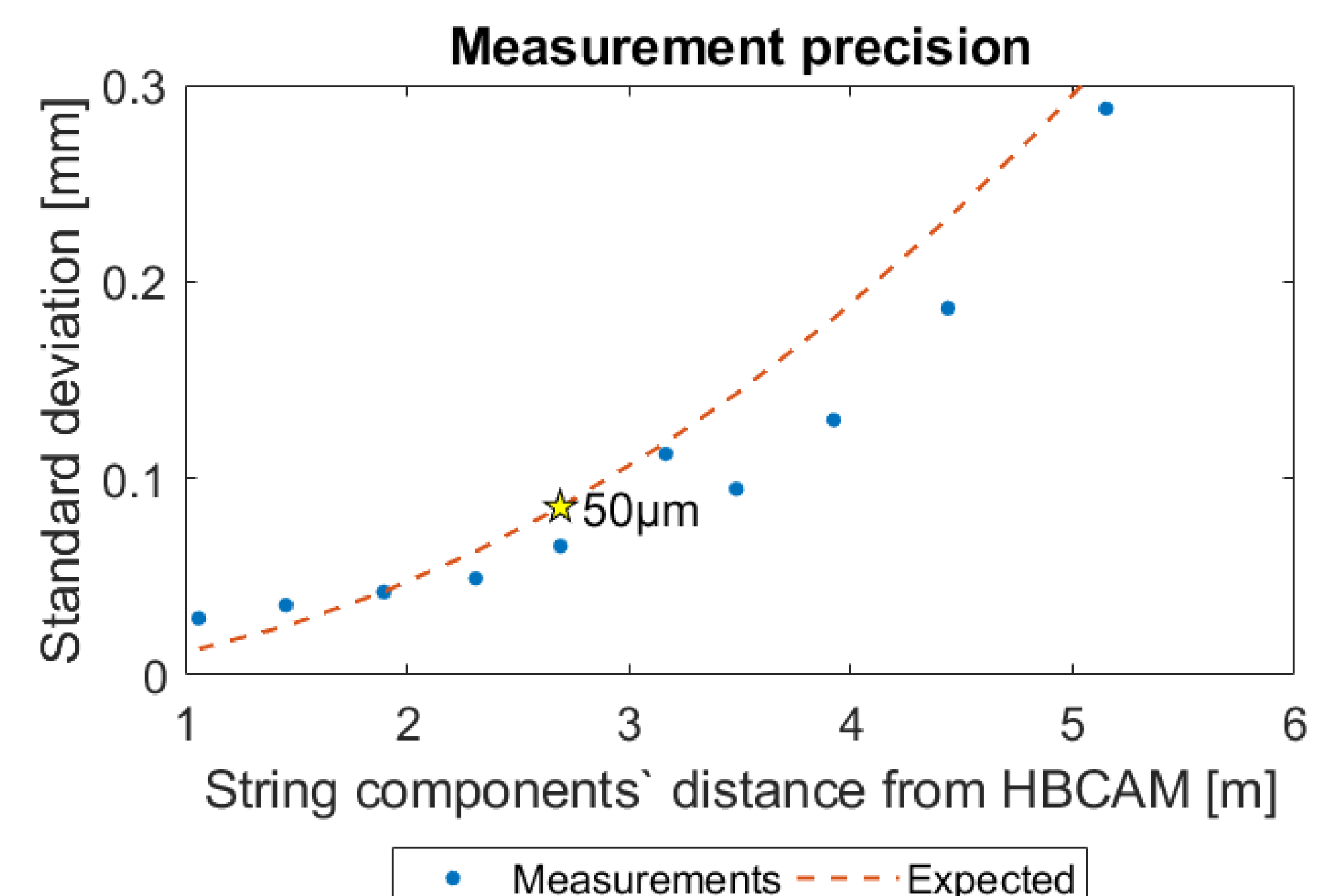

The measurement uncertainty for each component in the string assembly depends from the distance to the camera. Acquired data are matching with the expected precision.
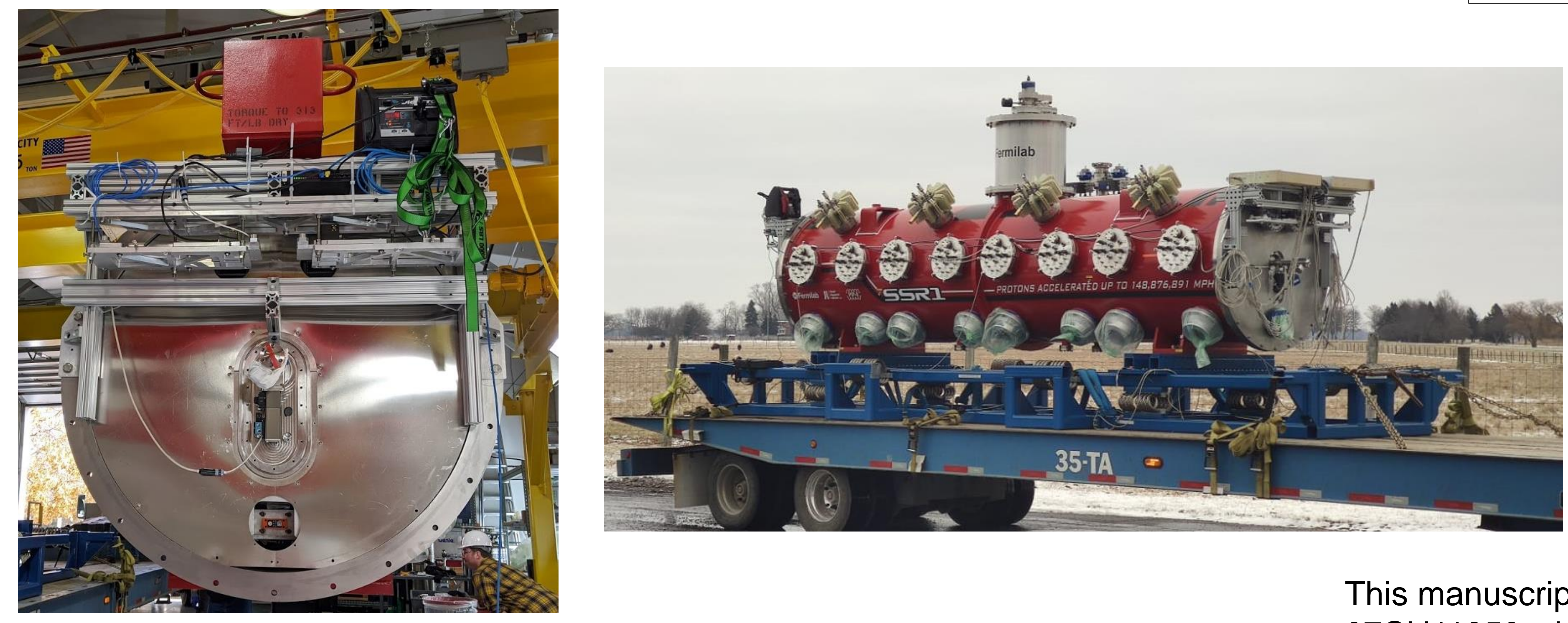

MONITORING DURING TRANSPORTATION

- The H-BCAMs are used also to monitor the position during the transportation from Lab-2 to ICB and from ICB to PIP2IT

- The results are cross-checked with accelerometers installed in the string assembly and the cryomodule

- Focus on a single cavity and monitor slow vibrations $<60 \mathrm{~Hz}$ 